\title{
Investigation of welds by the method of the magneto-optical eddy current flaw detection
}

\author{
Nazar Lugovskoy*, Vladimir Berzhansky, Dmitry Filippov, Anatoliy Prokopov, and Alexandr Shuyskyy \\ V.I. Vernadsky Crimean Federal University, 95007, Academician Vernadsky Ave. 4, Simferopol, Russia
}

\begin{abstract}
The paper deals with the magneto-optical eddy current method of flaw detection of conducting materials, in which epitaxial films of ferrite garnet are used as sensors. In particular, the possibilities of visualization of welded seams in magnetic and non-magnetic samples, as well as the defectoscopy of the seams themselves, are considered. The second part of the work is devoted to mathematical modeling of the distribution of the magnetic fields of eddy currents near similar defects.
\end{abstract}

\section{Experiment}

One of the prospective flaw detection technologies is the magneto-optical eddy current (MOEC) method, in which surface, subsurface and fatigue defects can be recorded in products from both magnetic and nonmagnetic metals [1-4].

\subsection{Introduction}

The principle of operation of the magneto-optical eddy current flaw detector is based on the reaction of the mobile domain structure (DS) of the sensor on the distribution of magnetic fields produced by the EC in the conductive material under study. The presence of defects in the metal object leads to a change in the trajectories of the eddy currents and to a corresponding change in the configuration of the magnetic fields generated by them. Visualization of the DS is carried out with the help of the Faraday Effect, as a result of which the rotation of the plane of polarization of the transmitted light occurs as a function of the direction of the local magnetization. The scheme and principle of operation are described in more detail in [3].

The main advantage of MOEC defectoscopy is the direct visualization of defects in real time, small dimensions and relative ease of manufacturing of the main parts of the flaw detector.

\subsection{Experimental technique and samples}

In $[3,4]$ the influence of different characteristics of the flaw detector, such as the frequency of the excitation current, the magnitude and sign of the acting magnetic fields, on the MOEC image was studied.

In this paper, we investigated the possibility of observing welds and hidden defects in them, in products made from magnetic and nonmagnetic metal alloys.
In our device we used alternating field inductors of two configurations that created a magnetic field either in the plane of the sample (parallel inductor) or normal to it (normal inductor).

During the measurements, single-crystal epitaxial films based on substituted bismuth ferrite-garnet with the general formula $(\mathrm{R}, \mathrm{Bi})_{3}(\mathrm{Fe}, \mathrm{M})_{5} 0_{12}$ [5] with the type of magnetic crystallographic anisotropy "easy axis" were used as a magneto-optical sensor. The thickness of the films varied in the range from 1.9 to $7.2 \mu \mathrm{m}$, the anisotropy field from 500 to $3000 \mathrm{Oe}$, the period of the domain structure from 5.5 to $32 \mu \mathrm{m}$, and the saturation field from 22 to 77 Oe.

\subsection{Non-defective welds}

Model non-defective welds made from both magnetic and non-magnetic materials were investigated. Figure 1 shows optical and magneto-optical images of welds in aluminum and stainless steel samples measured at a frequency $\mathrm{f}=25$ $\mathrm{kHz}$, alternating magnetic field $\mathrm{H}_{\mathrm{m}}=150$ Oe.

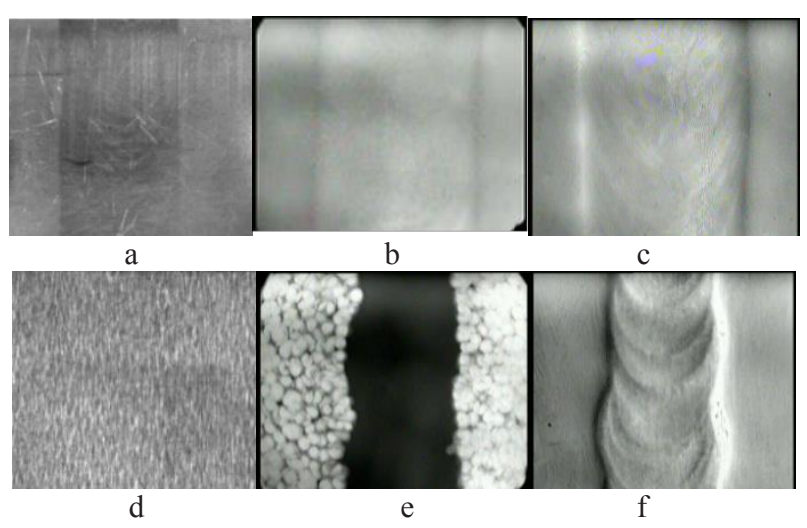

Fig. 1. Optical (a, d) and magneto-optical (b, c, e, f) images of welds: aluminum (a-c), stainless steel (d-f); orthogonal (b, e) and longitudinal (c, f) excitation of eddy currents.

It can be seen that welded seams are well registered in model samples of various types. In stainless steel samples,

* Corresponding author: lugovskoynv@mail.ru 
the sensitivity of the MO sensor is higher than in samples made of aluminum. Figure 1e shows a nonstationary cylindrical domain structure that fills the entire film space along the seam. The sensor above the seam is in a saturated state, which allows obtaining a clear and contrasting image of the welded seam. On MO images 1c and $1 \mathrm{f}$ we see an "analog picture" of the structure of the seam, especially on stainless steel, all the inhomogeneities (influxes) formed during the welding process and invisible during normal visual contact are visible. In the same pictures, we see clear welds boundaries represented by a black and white narrow frame of the defect. This indicates the opposite direction of the magnetic field of the eddy currents at the boundaries of the seam with longitudinal EC excitation.

The effect of the frequency of the alternating magnetic field on the MO imaging of the seams was investigated. It was found that the efficiency of MO imaging decreases with increasing frequency (Fig. 2) and to increase the efficiency it is required to increase the amplitude of the alternating magnetic field.

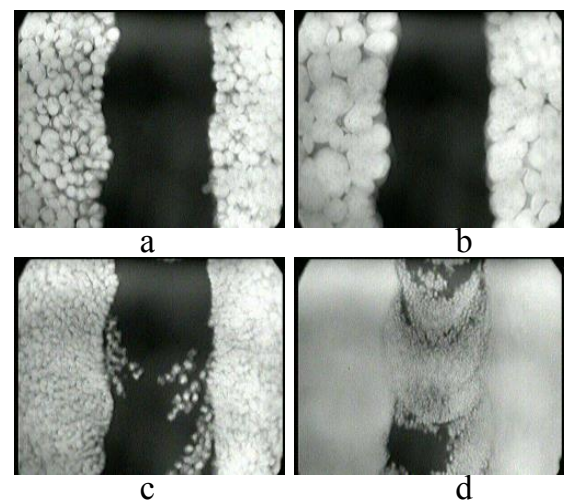

Fig. 2. Magnetooptical images of a weld in a stainless steel sample with orthogonal excitation of eddy currents with frequencies: a $-8, \mathrm{~b}-15, \mathrm{c}-30, \mathrm{~d}-45 \mathrm{kHz}$.

Analysis of MO images in magnetic and nonmagnetic samples shows that the magnitude of the EC magnetic fields is much larger in stainless steel samples. This is due to the much greater magnetic permeability of these samples. Therefore saturation of epitaxial ferrite-garnet films at the locations of defect mapping caused by EC magnetic fields occurs in a magnetic sample at lower values of $\mathrm{H}_{\mathrm{s}}=120$ Oe than in a nonmagnetic sample $\mathrm{H}_{\mathrm{s}}=$ 300 Oe. Such a state leads to a contrasting and clear image of defects. The use of the orthogonal constant bias field $\mathrm{H}_{-}$leads to a significant decrease of these values. For example, a constant bias field $\mathrm{H}_{-}=24$ Oe reduces saturation fields up to $80 \mathrm{Oe}$ and $220^{-} \mathrm{Oe}$ for magnetic and nonmagnetic samples, respectively. The effect of the constant bias field and the frequency of the alternating field on the MO imaging of defects in magnetic samples is more pronounced than in the nonmagnetic ones.

\subsection{Defective welds}

To test the operation of the MOEC method under real conditions, samples of welds of steel pipes with a diameter of 50 and $70 \mathrm{~mm}$ using electric and gas welding were made. MO defectoscopy was carried out at frequencies from 8 to $60 \mathrm{kHz}$, in alternating magnetic fields from 40 to 440 Oe. It was shown that the optimal conditions in this case are the following: an alternating current of $25 \mathrm{kHz}$, an alternating magnetic field of $80 \mathrm{Oe}$, a constant magnetic field of 0 Oe. Optical and MO images obtained under these conditions are shown in Figure 3.

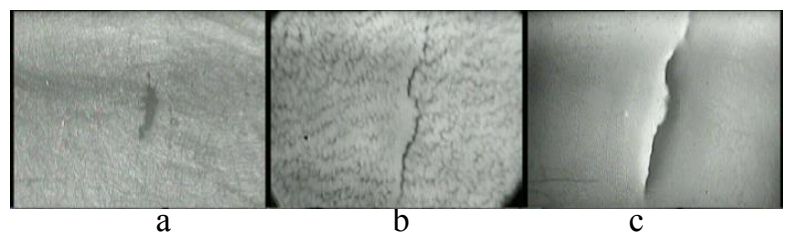

Fig. 3. The defects in welds of steel pipes: optical (a) and magneto-optical (b, c) images; orthogonal (b) and longitudinal (c) excitation of eddy currents.

MO images in Figure 3 were obtained with equal parameters of alternating and constant fields, the difference is only in the direction of generation of the excitation field. It is clearly seen that the MO image of the defective area contains much more information than a simple optical snapshot. The use of the MOEC method allows detecting defects that lie in the depth of the seam.

An interesting result was received the use of ferritegarnet film with an easy-plane anisotropy as a sensor. It made it possible to obtain an "analog picture" of the welds on a stainless steel sample using an inductor of a normal field.

\section{Simulation of magnetic field of eddy currents}

\subsection{Mathematic model}

A mathematical model of the magnetic field of a conducting block consisting of three parts with different values of electric conductivity and magnetic permeability is constructed. This mathematical model is a system of integro-differential equations for the densities of secondary sources. These sources include: eddy currents (determined by the instantaneous value of the bulk current density), electric charges (specified by the instantaneous value of the surface charge density), and magnetization currents (given by the instantaneous value of the surface current density). We note that the derivation of this system of integro-differential equations is carried out using the Ohm's law in differential form, as well as the boundary conditions for the normal component of the eddy current density and the tangential component of the magnetic field strength vector. [6]

The magnitude of the magnetic field acting on the magneto-optical sensor is superposition fields of the inductor and eddy current of the defect sample and can be represented in the form:

$$
\begin{aligned}
\vec{H}(M, t) & =\vec{H}_{0}(M, t)+\frac{1}{4 \pi \mu_{0}} \sum_{k=1}^{n} \mu_{k} \int_{V_{k}} \frac{\left[\vec{\delta}(N, t), \vec{r}_{M N}\right]}{r_{M N}^{3}} d V_{N}+ \\
& +\frac{1}{4 \pi} \sum_{k=1}^{m} \int_{S_{k}} \frac{\left[\vec{j}(P, t), \vec{r}_{M P}\right]}{r_{M P}^{3}} d S_{P}
\end{aligned}
$$


where $M$ - an arbitrary point of space (the point of observation); $N-$ point belonging to the volume of the conductor; $P-$ point belonging to the surface of a conductor; $\vec{H}_{0}(M, t)$ - the magnetic field of the inductor; $\vec{\delta}(N, t)$ - instantaneous value of the bulk density of eddy currents; $\vec{j}(P, t)$ - instantaneous value of the surface density of magnetization currents; $\mu_{k}$ magnetic permeability of the $k$ conductor; $n$ - number of volumes of conductors; $m$ - number of surfaces separating regions with different electrical conductivities.

\subsection{Non-ferromagnetic material}

We will give an example of modeling a magnetic field above the surface of a conducting massive block containing a defect in the form of a welded seam. First, consider the case where the block is made of a nonferromagnetic material (aluminum).

When manufacturing a welded seam, a change in the structure of the material inevitably occurs, which leads to a decrease in the conductivity of the conductor in the weld region. On figure 4 show are the distributions of the normal component of the intensity of the magnetic field in the region above the block.
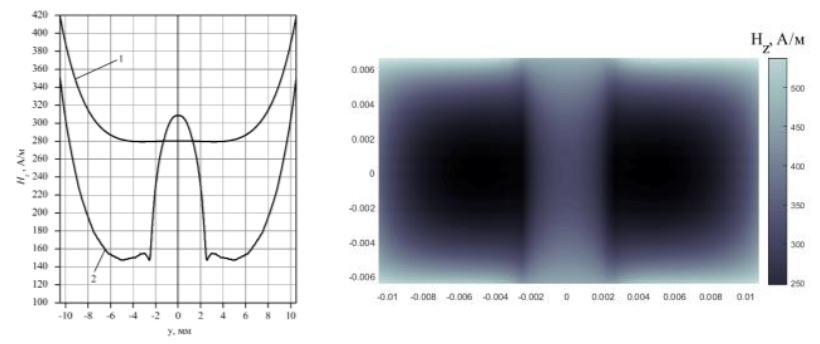

Fig.4. Distribution of strength magnetic field in the area above the block (a): block without weld (1); block with weld (2); topogram of the magnetic field in the region over a block with a welded seam (b).

In calculations, the conductivity of the weld was assumed to be $1.5 \times 10^{7}(\mathrm{~S} / \mathrm{m})$, which is $40 \%$ of the conductivity of pure aluminum. As can be seen, the presence of a weld with a lower conductivity than the conductivity of adjacent regions of the conductor leads to a significant increase in the contrast of the topogram of the magnetic field. The boundaries of the seam are clearly traced. It is natural to expect that the higher the difference in conductivity of are conductors, the more contrast the picture of the field, and, consequently, the MO image of varies part of sample will also be more contrast.

\subsection{Ferromagnetic material}

The presence of ferromagnetic properties in the sample from stainless steel leads to the appearance of magnetization currents. In contrast to eddy currents, the effect of which leads to a decrease in the resulting magnetic field, the magnetization currents tend to increase this field. Therefore, in the case of a non-ferromagnetic sample, the magnetic field is always smaller than in the case of a ferromagnetic sample, even with a relatively low magnetic permeability. The distribution of the normal component of the magnetic field, which allow one to compare the cases of a ferromagnetic and nonferromagnetic samples represented in figure 5 .

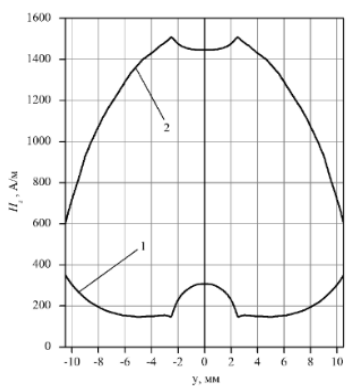

Fig. 5. Distribution of the normal component of the magnetic field intensity above the samples with a welded seam: nonferromagnetic block (1); ferromagnetic block (2).

The last figure shows that the field in the central region in the case of a ferromagnetic conductor exceeds the analogous value for a non-ferromagnetic conductor by more than 4.5 times. Note that in calculations it was assumed that the magnetic permeability of stainless steel is $30 \mu_{0}$. A significantly increased field in the case of the use of a ferromagnetic conductor can lead to a rapid saturation of the film, which is confirmed by the experimental results.

\section{Conclusions}

The possibility of using ferrite-garnet films to visualization and control the quality of defects of the "welded seam" type by the method of magneto-optical eddy current flaw detection has been shown.

The efficiency of visualization of welds seams in ferromagnetic samples is higher than in nonmagnetic samples and depends on the eddy current frequency and the constant bias field.

By the method of integro-differential equations, a model is obtained for the distribution of magnetic fields of eddy currents in the vicinity of welded seams, which is in satisfactory agreement with the experimental data.

\section{Acknowledgements}

The author Lugovskoy N.V. thanks for support the Project of Development Program of V.I. Vernadsky Crimean Federal University Network "Academic mobility of Young Scientists of Russia - AMMUR" in 2016

\section{References}

1. U. Radtke, R. Zielke, H. Rademacher, Opt. Laser. Eng. 36 (2001)

2. Y. Deng, X. Lin, Y. Fan, IEEE Trans. Magn. 42(2006)

3. V.Vishnevskiy, V.Berzhansky, N. Lugovskoy, A. Prokopov, F. Pankov, SSP, 230 (2014)

4. V.N. Berzhansky, D.M. Filippov, N.V. Lugovskoy, Phys. Procedia, 82C (2016)

5. V.N. Berzhansky, A.V. Karavainikov, T.V. Mikhailova, V.I. Belotelov, N.V. Lugovskoy, JMMM, 440 (2017)

6. D.M. Filippov, G.P. Kozik, A.V. Fursenko, V.N. Fedorovsky In Proc. ICIEAM 2 (2016) 